\title{
High Prevalence of Klebsiella pneumoniae Infections in AnHui Province: Clinical Characteristic and Antimicrobial Resistance
}

\author{
Cong Su $\mathbb{i}^{1, *}$ \\ Ting $\mathrm{Wu}^{2, *}$ \\ Bao Meng ${ }^{2}$ \\ Chengcheng Yue ${ }^{2}$ \\ Yating Sun ${ }^{2}$ \\ Lingling $\mathrm{He}^{2}$ \\ Tingting Bian ${ }^{2}$ \\ Yanyan Liu ${ }^{3,4}$ \\ Ying Huang ${ }^{5}$ \\ Yanhu Lan ${ }^{4}$ \\ Jiabin $\mathrm{Li}^{2-4,6}$
}

'Department of Infection Management, The First Affiliated Hospital of Anhui Medical University, Hefei, People's Republic of China; ${ }^{2}$ Department of Infectious Diseases, The First Affiliated Hospital of Anhui Medical University, Hefei, People's Republic of China; ${ }^{3}$ Anhui Center for Surveillance of Bacterial Resistance, Hefei, People's Republic of China; ${ }^{4}$ Institute of Bacterial Resistance, Anhui Medical University, Hefei, People's Republic of China; ${ }^{5}$ Department of Clinical Laboratory, The First Affiliated Hospital of Anhui Medical University, Hefei, People's Republic of China; ${ }^{6}$ Department of Infectious Diseases, The Chaohu Hospital of Anhui Medical University, Hefei, People's Republic of China

*These authors contributed equally to this work

Correspondence: Jiabin Li

Department of Infectious Diseases, The First Affiliated Hospital and the Chaohu Hospital of Anhui Medical University, Jixi Road 218 , Hefei, 230022, People's Republic of China Email lijiabin@ahmu.edu.cn

Yanhu Lan

Institute of Bacterial Resistance, Anhui Medical University, Jixi Road 218, Hefei, 230022, People's Republic of China

Email zixinhu@I63.com
Background: Klebsiella pneumoniae (K. pneumoniae) causes community-acquired and hospital-acquired pneumonia. The mortality rates of invasive infections caused by hypervirulent $K$. pneumoniae (HvKP) are extremely high. However, the microbiological characteristics and clinical manifestations of $K$. pneumoniae in AnHui province still remain unclear. Purpose: To show the high prevalence of HvKP infections regarding clinical characteristics and antimicrobial resistance in Anhui province.

Patients and Methods: A retrospective analysis was conducted to study the clinical data of 115 strains of K. pneumoniae from July 2019 to March 2020 in The First Affiliated Hospital of AnHui Medical University. The virulence genes, capsular types, carbapenemase genes, and molecular subtypes of these hypervirulent isolates were detected.

Results: Overall, 59.1\% (68/115) cases were HvKP infections, mainly from the department of intensive care unit (ICU, $n=14,20.6 \%$ ) and the department of respiratory and critical care $(n=13,19.1 \%)$. K2 was the most prevalent capsular serotype $(n=26)$, followed by K1 $(n=21)$. The results of MLST identification of 68 strains showed that ST23 ( $\mathrm{n}=15,22.1 \%)$ was the most common type of ST, followed by ST11 and ST65 (n=12, 17.6\%), ST86 ( $n=9,13.2 \%)$, and ST412 (n=6, 8.8\%). Among 68 hvKP strains, 12 isolates were carbapenem resistant, and all except two harboured $K P C$.

Conclusion: The high incidence of carbapenemase producing HvKP in the Anhui province, especially the higher mortality of HvKP, should be paid more attention. Meanwhile, epidemiological surveillance and clinical treatment strategies should be continuously determined and implemented.

Keywords: Klebsiella pneumoniae, virulence genes, capsular types, antimicrobial resistance

\section{Introduction}

Klebsiella pneumoniae was first isolated in 1893 by Friedlander, from the lung tissues of patients with lobar pneumonia. It is a Gram-negative bacterium with a thick capsule, and easily causes community and nosocomial infections. After the first report of hypervirulent K. pneumoniae (HvKP) by Chinese researchers in Taiwan in 1986, reports on HvKP have continued to emerge. In most cases, high mucilage often indicates that the bacteria have strong virulence. Unlike common $K$. pneumoniae, HvKP can arouse severe metastatic infections such as liver abscesses, endophthalmitis, and bacteraemia in young and healthy populations. Previous studies have demonstrated that $K$. pneumoniae rates were $5 \%$ among healthy Korean adults. ${ }^{1,2}$ Data from studies in areas where HvKP is endemic 
indicates that its prevalence can reach $12-45 \% .^{3-5}$ In China, the prevalence rates of HvKP infections are about $8.33-73.9 \%$, and the mortality rate is even as high as $60 \%{ }^{6-8}$ Colonization of the gastrointestinal system further promotes community transmission. ${ }^{9}$ Analyses of the Human Microbiome Project in the United States shows about $4 \%$ colonization rate for $K$. pneumoniae in faecal samples. ${ }^{10}$ However, the rate of $K$. pneumoniae colonization in hospitalized patients was $19-38 \% .^{11,12}$ At present, consistent with the string test positive for high viscosity is high appraisal main virulence $K$. pneumonia bacteria method, but it is still controversial that mucous is necessarily a high virulence. Therefore, it is inaccurate to define whether the strain is a highly virulent one only by positive "string test". 13,14 The virulence plasmid pLVPK, which carries the virulence genes $r m p A$ and $r m p A 2$, plays an important role in the virulence of HvKP. Therefore, when $\operatorname{rmp} A, \operatorname{rmp} A 2$, and string test were all positive, the strain is defined as HvKP. ${ }^{15}$ Our study also defined HvKP according to such criteria. In general, strains that are both highly virulent and resistant are rare. However, owing to the abuse of antibacterial drugs, a growing number of studies have reported the presence of multidrug-resistant (MDR) the carbapenemase producing HvKP, which is understandably detrimental. ${ }^{7,16-19}$ In this study, we collected 115 $K$. pneumoniae strains that were "string test" positive in the First Affiliated Hospital of Anhui Medical University from July 2019 to March 2020, and screened 68 strains of HvKP to further explore the molecular biological characteristics of HvKP and antimicrobial resistance to lay a foundation for subsequent scientific research and clinical treatment.

\section{Materials and Methods}

\section{Clinical K. pneumoniae Isolates and Patients' Data Collection}

We collected 115 consecutive patients at The First Affiliated Hospital of Anhui Medical University who were infected $K$. pneumoniae and showed positive string test. All specimens were numbered from 1 to 115 and stored at $-80^{\circ} \mathrm{C}$. Some clinical data and microbiological data related to the specimens could be obtained from the electronic or paper medical records of the hospital and the microbiological database. The inpatient information included demographic characteristics, clinical manifestations, microbiological reports, post-admission treatment, outcomes, and prognosis. Each patient in this study was admitted to the hospital after signing an informed consent.

\section{Determination of Hypervirulent Phenotype}

Phenotypic identification of $K$. pneumoniae relies on the classical "string test". 8 The strain taken from the refrigerator of $-80^{\circ} \mathrm{C}$ was inoculated on an agar plate and cultured overnight in the incubator at $37^{\circ} \mathrm{C}$ until colony formation. Using a bacterial inoculation ring for stretching, a positive string test that formed mucoviscous string $>5 \mathrm{~mm}$ was considered as the hypervirulent phenotype of $K$. pneumoniae.

\section{Capsular Serotyping and Determination of Virulence Genes rmpA, rmpA2}

All strains were grown overnight on agar plates, the genomic DNA was extracted. Then, polymerase chain reaction (PCR) was used to amplify virulence genes $r m p A$ and rmpA2 and capsular serotype genes as previously described. $^{20-22}$ The primer sequences used are shown in I note you uploaded the file 07_Jul_2020_63.xlsx. Please advise what this is, is this to be published with your manuscript or was this requested by the reviewer? 1 . The reaction mixture was prepared as follows: initial denaturation at $95^{\circ} \mathrm{C}$ for $3 \mathrm{~min}$; followed by 35 cycles of $95^{\circ} \mathrm{C}$ for $30 \mathrm{~s}, 52^{\circ} \mathrm{C}$ for $30 \mathrm{~s}$, and $72^{\circ} \mathrm{C}$ for $3 \mathrm{~min}$ and a final extension at $72^{\circ} \mathrm{C}$ for $10 \mathrm{~min}$. Agarose gel electrophoresis and sequencing were used to analyse the PCR products. The capsular serotypes of 12 strains carbapenemase producing HvKP were detected by wzi gene sequencing. The results were submitted to http://bigsdb.pasteur.fr.

\section{Susceptibility Testing and KPC Gene Identification}

The agar dilution method was used to identify and test antimicrobial susceptibility. The antimicrobial agents included ceftazidime, ceftriaxone, cefepime, cefotaxime, piperacillin-tazobactam, cefoperazone-sulbactam, imipenem, meropenem, amikacin, gentamicin, ciprofloxacin, levofloxacin, and aztreonam. Broth microdilution method was used for drug sensitivity tests of tigecycline and colistin (since there was no MIC breakpoints of these two drugs on CLSI, we referred to it of EScherichia coli on EUCAST). K. pneumoniae 700,603 was selected as the positive control group for antimicrobial susceptibility testing, and Escherichia coli 25,922 was used as the negative control. The Clinical and Laboratory Standards Institute 
Table I Primer Sequence About Virulence and Resistance Associated Genes

\begin{tabular}{|c|c|c|}
\hline \multicolumn{2}{|c|}{ Prime Name } & \multirow{2}{*}{$\frac{\text { Sequence }}{\text { ACTGGGCTACCTCTGCTTCA }}$} \\
\hline rmpA & Forward & \\
\hline & Reverse & CGCACCAGTAATTCCAACAG \\
\hline \multirow[t]{2}{*}{$r m p A 2$} & Forward & CTTTATGTGCAATAAGGATGTT \\
\hline & Reverse & ССTCCTGGAGAGTAAGCATT \\
\hline \multirow[t]{2}{*}{$\mathrm{KI}$} & Forward & GGTGCTCTTTACATCATTGC \\
\hline & Reverse & GCAATGGCCATTTGCGTTAG \\
\hline \multirow[t]{2}{*}{ K2 } & Forward & GGAGCCATTTGAATTCGGTG \\
\hline & Reverse & TCCCTAGCACTGGCTTAAGT \\
\hline \multirow[t]{2}{*}{ K5 } & Forward & GCCACCTCTAAGCATATAGC \\
\hline & Reverse & CGCACCAGTAATTCCAACAG \\
\hline \multirow[t]{2}{*}{ K20 } & Forward & CCGATTCGGTCAACTAGCTT \\
\hline & Reverse & GCACCTCTATGAACTTTCAG \\
\hline \multirow[t]{2}{*}{ K54 } & Forward & CATTAGCTCAGTGGTTGGCT \\
\hline & Reverse & GCTTGACAAACACCATAGCAG \\
\hline \multirow[t]{2}{*}{$K P C-2$} & Forward & ATGTCACTGTATCGCCGTCT \\
\hline & Reverse & TTTTCAGAGCCTTACTGCCC \\
\hline \multirow[t]{2}{*}{ NDM-I } & Forward & GGTTTGGCGATCTGGTTTTC \\
\hline & Reverse & CGGAATGGCTCATCACGATC \\
\hline \multirow[t]{2}{*}{ VIM-I } & Forward & AAATTCCGGTCGGAGAGGTC \\
\hline & Reverse & AATGCGCAGCACCAGGATAG \\
\hline \multirow[t]{2}{*}{ IMP-I } & Forward & GGAATAGAGTGGCTTAATTCTCC \\
\hline & Reverse & GGTTTAATAAAACAACCACC \\
\hline \multirow[t]{2}{*}{$O X A-48$} & Forward & GCGTGGTTAAGGATGAACAC \\
\hline & Reverse & CATCAAGTTCAACCCAACCG \\
\hline
\end{tabular}

(CLSI) guidelines and CLSI breakpoints or European Committee on Antimicrobial Susceptibility Testing criteria (version 10.0, http://www.eucast.org/clinicalbreakpoints/), respectively (CLSI 2021), can be referred to for specific experimental methods and result analysis. Moreover, Table 1 showed the identification of $K P C$ gene, including $K P C-2, N D M-1, V I M-1, I M P-1$ and $O X A-48$.

\section{Multilocus Sequence Typing (MLST)}

MLST typing was carried out for all HvKP strains screened according to the MLST website (http://www.pas
teur.fr/recherche/genopole/PF8/mlst/Kpneumoniae.html) with seven housekeeping genes (gapA, infB, $m d h$, phoE, pgi, rpoB, and tonB). We have submitted new alleles and STs that have not been previously reported.

\section{Pulsed-Field Gel Electrophoresis (PFGE)}

All isolated strains were subjected to PFGE. $X B a$ I (TaKaRa, Lot ${ }^{\#}$ AIF2232A) was used as the restriction endonuclease, and Salmonella H9812 as the marker for DNA size of PFGE electrophoresis. All strains were prepared by gelatinization, enzymatic digestion, and electrophoresis. Then, the PFGE images were processed by BioNumerics software and the tree diagram were drawn. The similarity coefficient of the strains in the similarity analysis matrix $>80 \%$ were of the same PFGE type. ${ }^{23}$

\section{Statistical Analysis}

Data analysis was performed using SPSS software (version 23.0; IBM Corporation, Armonk, NY, USA). If the continuous variables followed a normal distribution, they were indicated using mean \pm standard deviation (SD). Chisquare or Fisher exact tests were used to analyse categorical variables. When $P<0.05$, difference between the two groups was considered statistically significant.

\section{Results}

\section{HvKP Isolates Clinical Characteristics}

Among 115 strains, 59.1\% (68/115) isolates were HvKP, and $40.9 \%(47 / 115)$ were non-HvKP. Figure 1 shows the department source distribution of $68 \mathrm{HvKP}$ isolates: $20.6 \%(\mathrm{n}=14)$ were from the ICU and $19.1 \%(n=13)$ were from the Department of Respiratory and Critical Care. Table 2 summarizes the clinical characteristics of HvKP patients and nonHvKP patients. In the HvKP group, there were 43 (63.2\%) male and $25(36.8 \%)$ female patients. The mean age was $57.01 \pm 19.00$ years. Among all 68 community-acquired HvKP isolates, 41 patients (60.33\%) had pulmonary disease; 33 patients $(48.5 \%)$ were attached to invasive equipment; and $21(30.9 \%), 18(26.5 \%)$, and $8(11.8 \%)$ patients, respectively, had hypertension, diabetes, and liver abscess.

\section{Capsular Serotyping of HvKP and Non-HvKP}

Thus far, more than 100 serotypes of $K$. pneumoniae have been reported, most of which are closely associated with the types of infection and severity of disease progression. $^{24-26}$ Common clinical capsular serotypes 


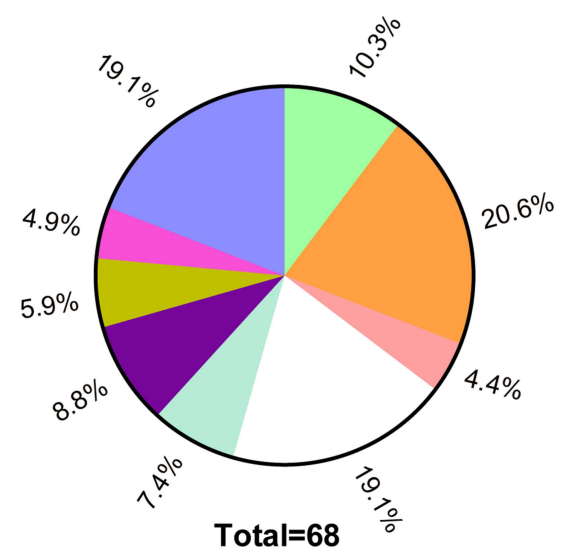

Figure I Distribution percentage of 68 strains of HvKP.

include $\mathrm{K} 1, \mathrm{~K} 2, \mathrm{~K} 5$, $\mathrm{K} 20$, and $\mathrm{K} 54$. We detected the capsular serotypes of the collected clinical isolates, and the results are shown in Table 2. Among HvKP isolates, the most common capsular serotype was K2 $(n=26$, $38.2 \%)$, followed by $\mathrm{K} 1 \quad(\mathrm{n}=21,30.9 \%)$. Among non$\mathrm{HvKP}$ isolates too, the most common serotype was $\mathrm{K} 2$ $(\mathrm{n}=9,19.1 \%)$, followed by K1 $(\mathrm{n}=2,4.3 \%)$. Obviously, both $\mathrm{K} 1$ and $\mathrm{K} 2$ serotypes were higher in HvKP than in non-HvKP $(P<0.05)$.

\section{Antimicrobial Resistance Between HvKP and Non-HvKP}

The sensitivity and drug resistance of 15 antimicrobial agents among HvKP and non-HvKP isolates are shown in Table 2. The resistance rate of cephalosporins in the non-HvKP group was higher than that of the HvKP group. All HvKP are sensitive to tigecycline and colistin. There were 23 carbapenemase producing isolates (12 strains in the HvKP group and 11 strains in the non-HvKP group) out of all 115 clinical strains.

\section{Prevalence of Carbapenemase Producing HvKP}

The demographic, microbiological, and clinical characteristics of the 12 carbapenemase producing HvKP patients are shown in Table 3. Nine patients developed pneumonia, and three patients experienced sepsis. Most of the 12 carbapenemase producing HvKP strains contained at least three genes encoding carbapenemase producing isolates. Consistent with domestic reports, ${ }^{27,28}$ the results of this study showed that ST11 was the dominant serotype in carbapenemase producing HvKP isolates.

\section{MLST Genotyping and PFGE}

MLST identification of 68 strains showed that ST23 $(n=15$, $22.1 \%$ ) was the most common type of ST, followed by ST11 and ST65 $(n=12,17.6 \%)$, ST86 $(n=9,13.2 \%)$, and ST412 $(n=6,8.8 \%)$. Homology analysis of the 68 strains of HvKP was conducted by PFGE. After $X B a \mathrm{I}$ digestion and BioNumerics software processing, a tree diagram was obtained (Figure 2). Briefly, 40 groups of different PFGE bands were detected in 68 strains of HvKP, indicating that the strain was highly polymorphic. Strain No. 32, 35, 37, and 38 of the 12 strains of carbapenemase producing HvKP were highly homologous, and most likely originated from the same strain or was caused by nosocomial infection.

\section{Discussion}

Klebsiella pneumoniae is one of the three most common causes of Gram-negative hospital-acquired infections (HAI, 10.2\%), second only to Pseudomonas aeruginosa $(11.5 \%)$ and Escherichia coli (10.4\%). ${ }^{29}$ Klebsiella pneumoniae infections can occur in any individual and thrive in different regions of the body. The mortality rate of bacteraemia caused by Klebsiella pneumoniae is about 20 $26 \%{ }^{30}$ This life-threatening bacterial infection has been emerging in many countries of the world and has become a major threat to host health. ${ }^{31}$

HvKP was defined on the basis of positive virulence genes rmpA and rmpA2 and a positive string test. As is known to all, $\sim 200 \mathrm{~kb}$ virulence plasmid is an important characteristic of HvKP, which contains many virulence coding genes, such as rmpA and rmpA2 mentioned above and siderophore (aerobactin and salmochelin). Nassif et al demonstrated that the aerobactin and myxoid phenotype 
Table 2 Clinical and Microbiological Characteristics of HvKP Isolates

\begin{tabular}{|c|c|c|c|}
\hline \multirow[t]{2}{*}{ Characteristics } & \multicolumn{2}{|c|}{ NO.(\%) of Isolates } & \multirow[t]{2}{*}{$P$ value } \\
\hline & $\begin{array}{l}\text { HvKP } \\
(n=68)\end{array}$ & $\begin{array}{c}\text { Non-HvKP } \\
(n=47)\end{array}$ & \\
\hline \multicolumn{4}{|c|}{ Demographic characteristics } \\
\hline Male sex & $43(63.2)$ & $35(74.5)$ & 0.205 \\
\hline $\begin{array}{l}\text { Age (years) (mean } \\
\pm \mathrm{SD})\end{array}$ & $57.01 \pm 19.00$ & $57.26 \pm 16.20$ & 0.944 \\
\hline \multicolumn{4}{|l|}{ K serotypes } \\
\hline $\mathrm{KI}$ & $21 / 68(30.9)$ & $2 / 47(4.3)$ & 0.000 \\
\hline $\mathrm{K} 2$ & $26 / 68(38.2)$ & $9 / 47(19.1)$ & 0.029 \\
\hline K5 & $12 / 68(17.6)$ & $4 / 47(8.5)$ & 0.164 \\
\hline $\mathrm{K} 20$ & $0 / 68(0.0)$ & 2/47 (4.3) & 0.086 \\
\hline K57 & $8 / 68(11.8)$ & $\mathrm{I} / 47(2.1)$ & 0.059 \\
\hline \multicolumn{4}{|c|}{ Antimicrobial susceptibility } \\
\hline Ceftazidime & 13/68 (19.1) & $12 / 47(25.5)$ & 0.093 \\
\hline Ceftriaxone & 14/68 (20.6) & I5/47 (32.0) & 0.028 \\
\hline Cefepime & $13 / 68(19.1)$ & $11 / 47(23.4)$ & 0.030 \\
\hline Cefotaxime & 14/68 (20.6) & $15 / 47(32.0)$ & 0.028 \\
\hline $\begin{array}{l}\text { Piperacillin- } \\
\text { tazobactam }\end{array}$ & $12 / 68(17.6)$ & $9 / 47(19.1)$ & 0.273 \\
\hline $\begin{array}{l}\text { Cefoperazone- } \\
\text { sulbactam }\end{array}$ & 13/68 (19.1) & 10/47 (21.3) & 0.254 \\
\hline Imipenem & 12/68 (17.6) & 10/47 (21.3) & 0.168 \\
\hline Meropenem & $12 / 68(17.6)$ & $10 / 47(21.3)$ & 0.168 \\
\hline Amikacin & $12 / 68(17.6)$ & $6 / 47(12.8)$ & 0.872 \\
\hline Gentamicin & $13 / 68(19.1)$ & 10/47 (21.3) & 0.254 \\
\hline Ciprofloxacin & 15/68 (22.1) & I4/47 (29.8) & 0.082 \\
\hline Levofloxacin & 14/68 (20.6) & 13/47 (27.7) & 0.088 \\
\hline Aztreonam & 15/68 (22.1) & I4/47 (29.8) & 0.082 \\
\hline Tigecycline & $0 / 68(0)$ & - & - \\
\hline Colistin & $0 / 68(0)$ & - & - \\
\hline Carbapenemase & $12 / 68(17.6)$ & I I/47 (23.4) & 0.448 \\
\hline \multicolumn{4}{|l|}{ Diseases } \\
\hline Cancer & $7(10.3)$ & $12(25.5)$ & 0.031 \\
\hline Liver abscess & $8(I I .8)$ & I $(0.02)$ & 0.059 \\
\hline Hypertension & $21(44.7)$ & $15(32.0)$ & 0.907 \\
\hline Diabetes & $18(26.5)$ & $8(17.0)$ & 0.234 \\
\hline Pulmonary disease & $19(28.0)$ & $12(25.5)$ & 0.775 \\
\hline Invasive equipment & $33(48.5)$ & $21(44.7)$ & 0.684 \\
\hline
\end{tabular}

were associated with type $\mathrm{K} 1$ and $\mathrm{K} 2 .{ }^{32,33}$ Ye et al found that all detected strains contained iuc, iro, rmpA and rтpA2 genes in their study of 40 pyogenic liver abscess specimens. ${ }^{34}$ RmpA/rmpA2 gene and siderophores cluster are considered to be more important in invasive infections caused by Klebsiella pneumoniae. ${ }^{35}$ Some studies have found that $i r o B, i u c A$, peg-344, rmpA, and $r m p A 2$ are the most accurate molecular markers for differentiating between HvKP and classical Kp strains. ${ }^{36}$ Indeed, in addition to rmpA and rmpA2, iucA (encoding aerobactin) has been demonstrated to be one of the most accurate genetic markers for identifying HvKP and has not been studied in the present work.

The top three units from where the $68 \mathrm{HvKP}$ strains were isolated were the Department of ICU ( $n=14,20.6 \%)$, Department of Respiratory Medicine and Critical Care $(\mathrm{n}=13,19.1 \%)$, and the Department of Infectious Disease $(n=7,10.3 \%)$, poor physical quality and low immunity of the patient. Thus, these departments should be paid more attention to, to control HvKP infections. Consistent with previous studies, our results show that neither age nor sex is associated with HvKP. ${ }^{37}$ However, K. pneumoniae is highly aggressive and has been linked to infections in healthy young people. ${ }^{38-41}$ Studies have shown that HvKP infection is more prone to metastatic infection than non-HvKP infections. ${ }^{42,43}$ But our study shows that patients with non-HvKP infection are more susceptible to tumours than those who are infected with HvKP $(25.5 \%$ vs $10.3 \%, p=0.031$ ). Other diseases or invasive equipment possibly have no association with HvKP.

Our study showed that HvKP was associated with K1 and $\mathrm{K} 2$ expression ( $p<0.05$, Table 2$)$, which is consistent with previous research. ${ }^{8}$ Liu et al suggested that the resistance of $\mathrm{K} 1$ and $\mathrm{K} 2$ capsular serotypes to phagocytosis might be one of the causes, thus providing favourable conditions for the colonization and growth of bacteria. ${ }^{22}$ Different from other literature reports, ${ }^{44}$ our results showed that the most common capsular serotype in HvKP strain was K2 $(26 / 68,38.2 \%)$, followed by K1 $(21 / 68,30.9 \%)$. However, in general, these two serotypes are predominant.

Carbapenems including imipenem, meropenem, and ertapenem are currently considered the most effective antibiotics in the treatment of Gram-negative bacilli infection. However, owing to the abuse of antibacterial drugs, especially carbapenem antibiotics, multidrug-resistant $K$. pneumoniae strains are constantly emerging, making it difficult to control these infections. ${ }^{45}$ The high virulence and antimicrobial resistance of $K$. pneumoniae vary to a large extent. ${ }^{46}$ In our study, 12 of the 23 carbapenemase producing strains were HvKP isolates, and 3 of these 12 individuals eventually died. The differences in carbapenemase producing isolates between the HvKP and non-HvKP group showed no statistical significance $(17.6 \%$ vs $23.4 \%, p=0.448)$. We speculate that this is because of the 


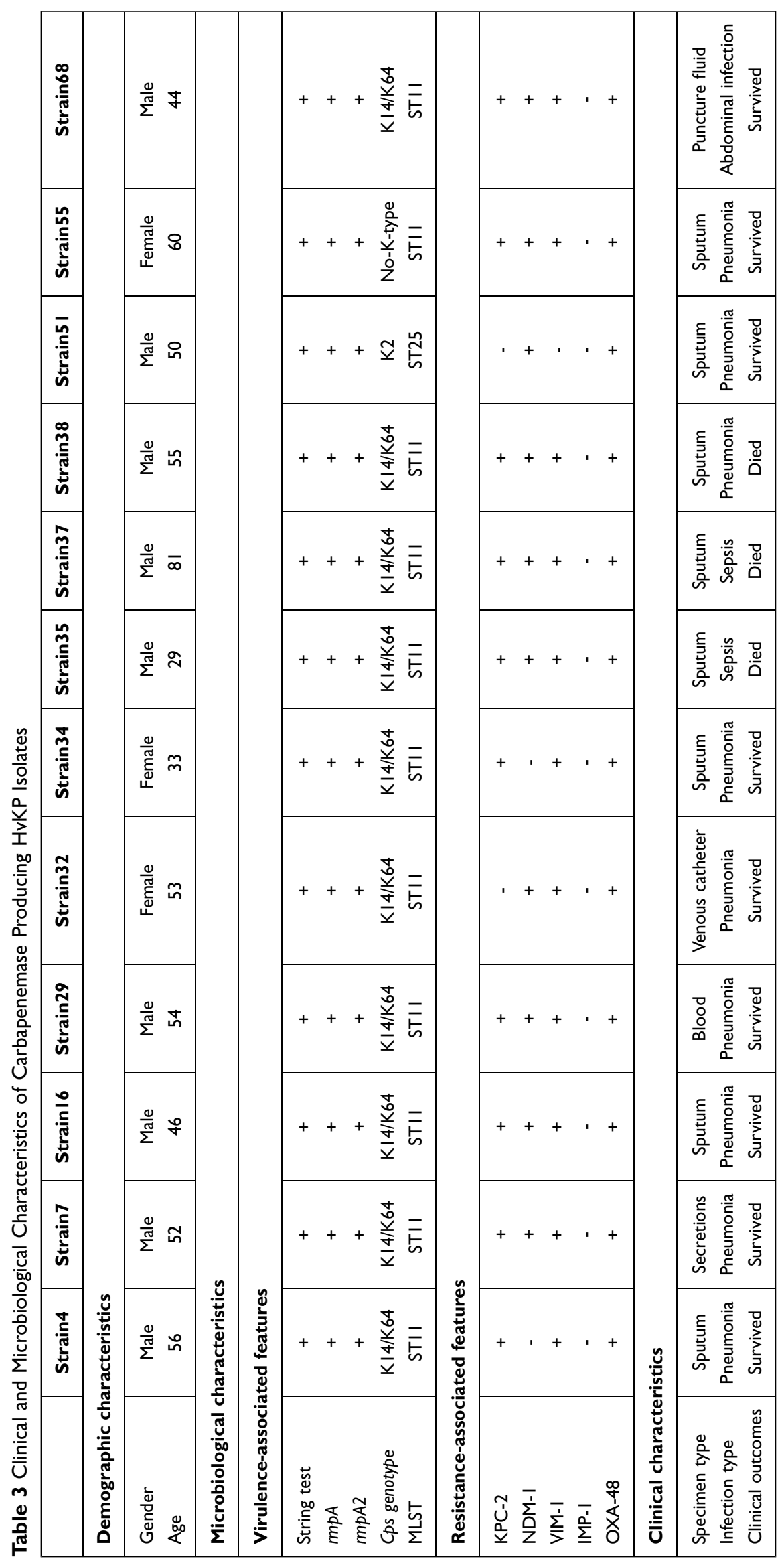




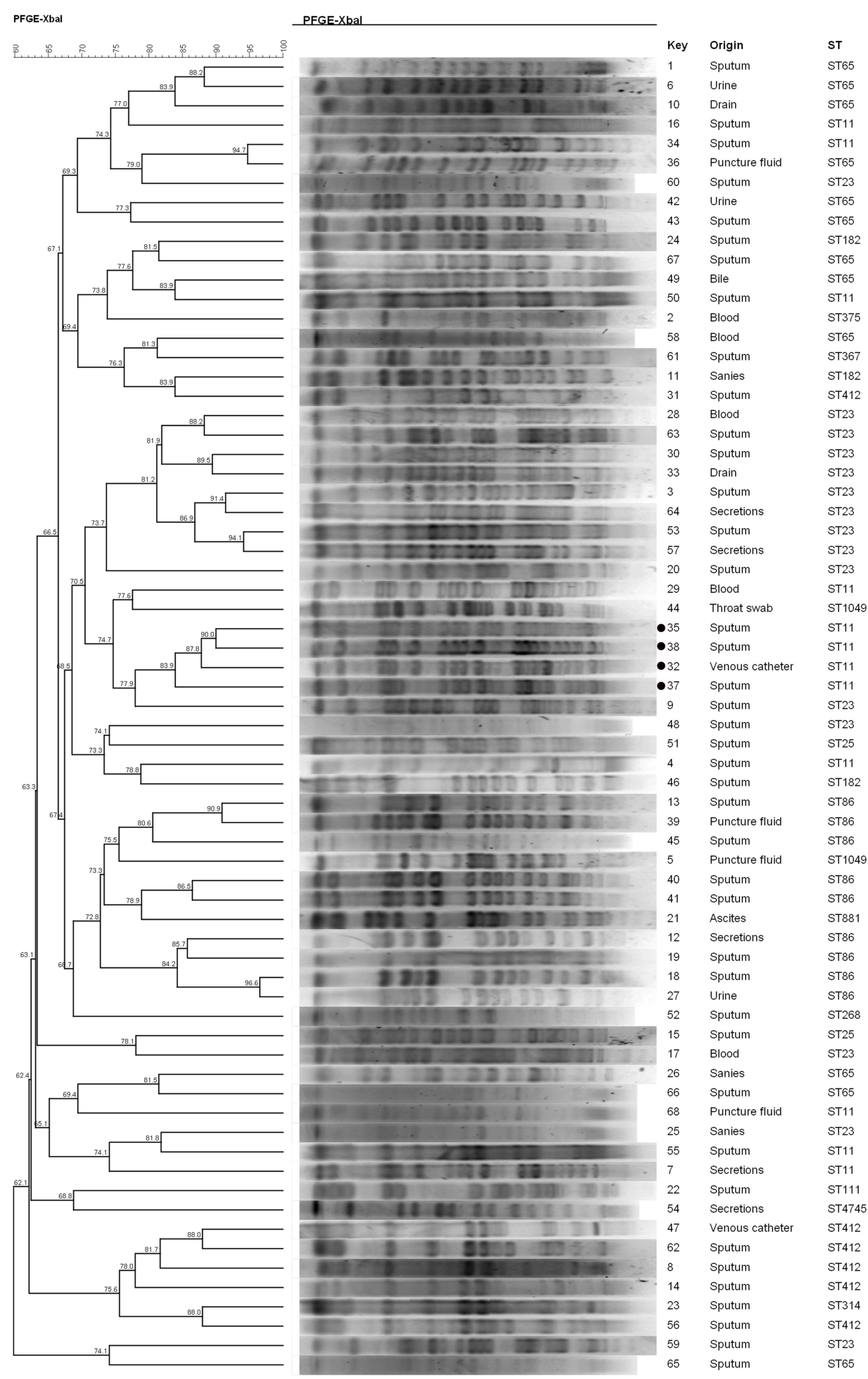

Figure 2 Pulsed field gel electrophoresis (PFGE) cluster analysis of 68 strains of HvKP from different sources. Four highly homologous isolates from carbapenemase producing HvKP have been marked with a black circle to the serial number left. 
insufficient number of clinical specimens. Regarding the rare co-existence of high virulence and resistance of $K$. pneumoniae, some scholars ${ }^{47}$ consider that HvKP has no resistance plasmids or that the resistance genes vanish when carrying virulence-related plasmids and genes. Further research studies are needed to verify these suggestions. The distribution and prevalence of ST types vary greatly from region to region. For example, ST23 is the most prevalent in Wuhan, accounting for $21.7 \%$, while ST11 is the most prevalent in Zhejiang, accounting for $25 \%{ }^{8}$ In our study, 11 of the 12 carbapenemase producing HvKP strains were ST11 clones, which indicated that this strain might have an epidemic phenomenon over a certain period of time. The source and collection time of these strains were variable, and the circulation of hospital personnel or airborne transmission further increased the possibility of infections in patients. Therefore, hand hygiene of medical staff and infection prevention and control in hospitals are paramount.

Carbapenemase producing HvKP has caused fatal infections, ${ }^{16,19}$ which requires immediate action in case of multidrug-resistant infections. However, there are few studies on this strain in Anhui province. Our research highlights the high prevalence of HvKP infections in the Anhui province, including clinical characteristics and antimicrobial resistance. These severe infections caused by HvKP and the continuous occurrence of carbapenemase producing HvKP nudge us to improve clinical awareness, infection prevention, and treatment strategies.

\section{Conclusion}

Klebsiella pneumoniae (K. pneumoniae) causes community-acquired and hospital-acquired pneumonia. Our research shows the microbiological characteristics and clinical manifestations of $K$. pneumoniae in AnHui province, and the high incidence of carbapenemase producing HvKP, emphasizing the importance of monitoring, prevention and identifying clinical treatment strategies.

\section{Data Sharing Statement}

Data can be made available through contact with the corresponding author (Professor Jiabin Li).

\section{Ethics Approval and Informed Consent}

This study was conducted in accordance with the Declaration of Helsinki, and the protocols applied in this study were approved by the Ethics Committee of the First Affiliated Hospital of Anhui Medical University, China.

\section{Acknowledgments}

We sincerely thank Zhou Liu (Clinical Laboratory of the Second Affiliated Hospital of Anhui Medical University) and Kaili Sun (The First Affiliated Hospital of University of Science and Technology of China) for technical support.

\section{Funding}

This study was supported by the National Natural Science Foundation of China (grant no. 81973983), the National Science and Technology Major Project (grant no. 2017ZX10204401), the Borrowing and Transferring Subsidy Project in 2019, Hefei (grant no. J2019Y04), the Collaborative Tackling and Public Health Collaborative Innovation Project in Anhui Province (grant no. GXXT2020-018), the Joint Construction Project of Clinical Medicine University and Hospital (grant no. 2021lcxk006), and the Natural Science Research Project of Universities in Anhui Province (grant no. KJ2020A0176).

\section{Disclosure}

The authors declare no conflicts of interest in this work.

\section{References}

1. Chung DR, Lee H, Park MH, et al. Fecal carriage of serotype K1 Klebsiella pneumoniae ST23 strains closely related to liver abscess isolates in Koreans living in Korea. Eur J Clin Microbiol Infect Dis. 2012;31(4):481-486. doi:10.1007/s10096-011-1334-7

2. Lin YT, Siu LK, Lin JC, et al. Seroepidemiology of Klebsiella pneumoniae colonizing the intestinal tract of healthy Chinese and overseas Chinese adults in Asian countries. BMC Microbiol. 2012;12:13. doi:10.1186/1471-2180-12-13

3. Liu Z, Gu Y, Li X, et al. Identification and Characterization of NDM-1-producing Hypervirulent (Hypermucoviscous) Klebsiella pneumoniae in China. Ann Lab Med. 2019;39(2):167-175. doi:10.3343/alm.2019.39.2.167

4. Lan Y, Zhou M, Jian Z, et al. Prevalence of pks gene cluster and characteristics of Klebsiella pneumoniae-induced bloodstream infections. J Clin Lab Anal. 2019;33(4):e22838. doi:10.1002/ jcla. 22838

5. Liu C, Shi J, Guo J. High prevalence of hypervirulent Klebsiella pneumoniae infection in the genetic background of elderly patients in two teaching hospitals in China. Infect Drug Resist. 2018;11:1031-1041. doi:10.2147/IDR.S161075

6. Hu Y, Ping Y, Li L, et al. A retrospective study of risk factors for carbapenem-resistant Klebsiella pneumoniae acquisition among ICU patients. J Infect Dev Ctries. 2016;10(3):208-213. doi:10.3855/ jidc. 6697

7. Zhang Y, Zeng J, Liu W, et al. Emergence of a hypervirulent carbapenem-resistant Klebsiella pneumoniae isolate from clinical infections in China. J Infect. 2015;71(5):553-560. doi:10.1016/j. jinf.2015.07.010 
8. Zhang Y, Zhao C, Wang Q, et al. High prevalence of hypervirulent Klebsiella pneumoniae infection in China: geographic distribution, clinical characteristics, and antimicrobial resistance. Antimicrob Agents Chemother. 2016;60(10):6115-6120. doi:10.1128/AAC.01127-16

9. Choby JE, Howard-Anderson J, Weiss DS. Hypervirulent Klebsiella pneumoniae - clinical and molecular perspectives. J Intern Med. 2020;287(3):283-300. doi:10.1111/joim.13007

10. Conlan S, Kong HH, Segre JA. Species-level analysis of DNA sequence data from the NIH human microbiome project. PLoS One. 2012;7(10):e47075. doi:10.1371/journal.pone.0047075

11. Gorrie CL, Mirceta M, Wick RR, et al. Gastrointestinal carriage is a major reservoir of Klebsiella pneumoniae infection in intensive care patients. Clin Infect Dis. 2017;65(2):208-215. doi:10.1093/cid/ cix 270

12. Martin RM, Cao J, Brisse S, et al. Molecular epidemiology of colonizing and infecting isolates of Klebsiella pneumoniae. Msphere. 2016;1(5). doi:10.1128/mSphere.00261-16

13. Russo TA, Olson R, Macdonald U, et al. Aerobactin mediates virulence and accounts for increased siderophore production under iron-limiting conditions by hypervirulent (hypermucoviscous) Klebsiella pneumoniae. Infect Immun. 2014;82(6):2356-2367. doi:10.1128/IAI.01667-13

14. Alcantar-Curiel MD, Giron JA. Klebsiella pneumoniae and the pyogenic liver abscess: implications and association of the presence of rpmA genes and expression of hypermucoviscosity. Virulence. 2015;6 (5):407-409. doi:10.1080/21505594.2015.1030101

15. Chen YT, Chang HY, Lai YC, et al. Sequencing and analysis of the large virulence plasmid pLVPK of Klebsiella pneumoniae CG43. Gene. 2004;337:189-198. doi:10.1016/j.gene.2004.05.008

16. Zhang R, Lin D, Chan EW, et al. Emergence of carbapenem-resistant serotype K1 hypervirulent Klebsiella pneumoniae strains in China. Antimicrob Agents Chemother. 2016;60(1):709-711. doi:10.1128/ AAC.02173-15

17. $\mathrm{Xu} \mathrm{M,} \mathrm{Fu} \mathrm{Y,} \mathrm{Fang} \mathrm{Y,} \mathrm{et} \mathrm{al.} \mathrm{High} \mathrm{prevalence} \mathrm{of} \mathrm{KPC-2-producing}$ hypervirulent Klebsiella pneumoniae causing meningitis in Eastern China. Infect Drug Resist. 2019;12:641-653. doi:10.2147/IDR. S191892

18. Pan H, Lou Y, Zeng L, et al. Infections caused by carbapenemase-producing Klebsiella pneumoniae: microbiological characteristics and risk factors. Microb Drug Resist. 2019;25 (2):287-296. doi:10.1089/mdr.2018.0339

19. Liu Y, Li XY, Wan LG, et al. Virulence and transfer ability of resistance determinants in a novel Klebsiella pneumoniae sequence type 1137 in China. Microb Drug Resist. 2014;20(2):150-155. doi:10.1089/mdr.2013.0107

20. Fang CT, Lai SY, Yi WC, et al. Klebsiella pneumoniae genotype K1: an emerging pathogen that causes septic ocular or central nervous system complications from pyogenic liver abscess. Clin Infect Dis. 2007;45(3):284-293. doi:10.1086/519262

21. Compain F, Babosan A, Brisse S, et al. Multiplex PCR for detection of seven virulence factors and K1/K2 capsular serotypes of Klebsiella pneumoniae. J Clin Microbiol. 2014;52(12):4377-4380. doi:10.1128/ JCM.02316-14

22. Lin JC, Koh TH, Lee N, et al. Genotypes and virulence in serotype K2 Klebsiella pneumoniae from liver abscess and non-infectious carriers in Hong Kong, Singapore and Taiwan. Gut Pathog. 2014;6:21. doi:10.1186/1757-4749-6-21

23. $\mathrm{Ku} \mathrm{YH,} \mathrm{Chuang} \mathrm{YC,} \mathrm{Chen} \mathrm{CC,} \mathrm{et} \mathrm{al.} \mathrm{Klebsiella} \mathrm{pneumoniae} \mathrm{isolates}$ from meningitis: epidemiology, virulence and antibiotic resistance. Sci Rep. 2017;7(1):6634. doi:10.1038/s41598-017-06878-6

24. Wyres KL, Wick RR, Gorrie C, et al. Identification of Klebsiella capsule synthesis loci from whole genome data. Microb Genom. 2016;2(12):e000102. doi:10.1099/mgen.0.000102

25. Rafat C, Messika J, Barnaud G, et al. Hypervirulent Klebsiella pneumoniae, a 5-year study in a French ICU. J Med Microbiol. 2018;67(8):1083-1089. doi:10.1099/jmm.0.000788
26. Catalan-Najera JC, Barrios-Camacho H, Duran-Bedolla J, et al. Molecular characterization and pathogenicity determination of hypervirulent Klebsiella pneumoniae clinical isolates serotype K2 in Mexico. Diagn Microbiol Infect Dis. 2019;94(3):316-319. doi:10.1016/j.diagmicrobio.2019.01.013

27. Ho PL, Cheung YY, Wang Y, et al. Characterization of carbapenem-resistant Escherichia coli and Klebsiella pneumoniae from a healthcare region in Hong Kong. Eur $J$ Clin Microbiol Infect Dis. 2016;35(3):379-385. doi:10.1007/s10096-015-2550-3

28. Sun K, Chen X, Li C, et al. Clonal dissemination of multilocus sequence type $11 \mathrm{Klebsiella}$ pneumoniae carbapenemase - producing K. pneumoniae in a Chinese teaching hospital. Apmis. 2015;123 (2):123-127. doi:10.1111/apm.12313

29. Cai Y, Venkatachalam I, Tee NW, et al. Prevalence of healthcare-associated infections and antimicrobial use among adult inpatients in Singapore acute-care hospitals: results from the first national point prevalence survey. Clin Infect Dis. 2017;64(suppl_2): S61-S67. doi:10.1093/cid/cix103

30. Tan TY, Ong M, Cheng Y, Ng L. Hypermucoviscosity, rmpA, and aerobactin are associated with community-acquired Klebsiella pneumoniae bacteremic isolates causing liver abscess in Singapore. J Microbiol Immunol Infect. 2019;52(1):30-34. doi:10.1016/j.jmii.2017.07.003

31. Struve C, Roe CC, Stegger M, et al. Mapping the evolution of hypervirulent Klebsiella pneumoniae. Mbio. 2015;6(4):e00630. doi:10.1128/mBio.00630-15

32. Nassif X, Sansonetti PJ. Correlation of the virulence of Klebsiella pneumoniae $\mathrm{K} 1$ and $\mathrm{K} 2$ with the presence of a plasmid encoding aerobactin. Infect Immun. 1986;54(3):603-608. doi:10.1128/ iai.54.3.603-608.1986

33. Nassif X, Fournier JM, Arondel J, Sansonetti PJ. Mucoid phenotype of Klebsiella pneumoniae is a plasmid-encoded virulence factor. Infect Immun. 1989;57(2):546-552. doi:10.1128/iai.57.2.546-552.1989

34. Ye M, Tu J, Jiang J, et al. Clinical and genomic analysis of liver abscess-causing Klebsiella pneumoniae identifies new liver abscess-associated virulence genes. Front Cell Infect Mi. 201 $6 ; 6: 165$.

35. Holt KE, Wertheim H, Zadoks RN, et al. Genomic analysis of diversity, population structure, virulence, and antimicrobial resistance inKlebsiella pneumoniae, an urgent threat to public health. Proc Natl Acad Sci. 2015;112(27):E3574-E3581. doi:10.1073/pnas.1501049112

36. Russo TA, Olson R, Fang C-T, et al. Identification of biomarkers for differentiation of Hypervirulent Klebsiella pneumoniae from Classical K. pneumoniae. J Clin Microbiol. 2018;56(9). doi:10.112 8/JCM.00776-18

37. Yang Z, Liu W, Cui Q, et al. Prevalence and detection of Stenotrophomonas maltophilia carrying metallo-beta-lactamase blaL1 in Beijing, China. Front Microbiol. 2014;5:692. doi:10.3389/ fmicb.2014.00692

38. Lin YT, Jeng YY, Chen TL, Fung CP. Bacteremic communityacquired pneumonia due to Klebsiella pneumoniae: clinical and microbiological characteristics in Taiwan, 2001-2008. BMC Infect Dis. 2010;10:307. doi:10.1186/1471-2334-10-307

39. Pomakova DK, Hsiao CB, Beanan JM, et al. Clinical and phenotypic differences between classic and hypervirulent Klebsiella pneumonia: an emerging and under-recognized pathogenic variant. Eur J Clin Microbiol Infect Dis. 2012;31(6):981-989. doi:10.1007/s10096-0111396-6

40. Jung SW, Chae HJ, Park YJ, et al. Microbiological and clinical characteristics of bacteraemia caused by the hypermucoviscosity phenotype of Klebsiella pneumoniae in Korea. Epidemiol Infect. 2013;141(2):334-340. doi:10.1017/S0950268812000933

41. Brisse S, Fevre C, Passet V, et al. Virulent clones of Klebsiella pneumoniae: identification and evolutionary scenario based on genomic and phenotypic characterization. PLoS One. 2009;4(3):e4982. doi:10.1371/journal.pone.0004982 
42. Lin YT, Huang YW, Huang $\mathrm{HH}$, et al. In vivo evolution of tigecycline-non-susceptible Klebsiella pneumoniae strains in patients: relationship between virulence and resistance. Int $J$ Antimicrob Agents. 2016;48(5):485-491. doi:10.1016/j.ijantimicag.2016.07.008

43. Keynan Y, Karlowsky JA, Walus T, Rubinstein E. Pyogenic liver abscess caused by hypermucoviscous Klebsiella pneumoniae. Scand J Infect Dis. 2007;39(9):828-830. doi:10.1080/00365540701266763

44. Hao Z, Duan J, Liu L, et al. Prevalence of community-acquired, hypervirulent Klebsiella pneumoniae isolates in Wenzhou, China. Microb Drug Resist. 2020;26(1):21-27. doi:10.1089/mdr.2019.0096
45. Nordmann P, Naas T, Poirel L. Global spread of Carbapenemase-producing Enterobacteriaceae. Emerg Infect Dis. 2011;17(10):1791-1798. doi:10.3201/eid1710.110655

46. Bialek-Davenet S, Criscuolo A, Ailloud F, et al. Genomic definition of hypervirulent and multidrug-resistant Klebsiella pneumoniae clonal groups. Emerg Infect Dis. 2014;20(11):1812-1820. doi:10.3201/ eid2011.140206

47. Li W, Sun G, Yu Y, et al. Increasing occurrence of antimicrobial-resistant hypervirulent (hypermucoviscous) Klebsiella pneumoniae isolates in China. Clin Infect Dis. 2014;58(2):225-232. doi:10.1093/cid/cit675

\section{Publish your work in this journal}

Infection and Drug Resistance is an international, peer-reviewed openaccess journal that focuses on the optimal treatment of infection (bacterial, fungal and viral) and the development and institution of preventive strategies to minimize the development and spread of resistance. The journal is specifically concerned with the epidemiology of antibiotic resistance and the mechanisms of resistance development and diffusion in both hospitals and the community. The manuscript management system is completely online and includes a very quick and fair peerreview system, which is all easy to use. Visit http://www.dovepress.com/ testimonials.php to read real quotes from published authors. 